\title{
|Hospital Regional Materno Infantil de Imperatriz, Maranhão: via de parto predominante em outubro e novembro de 2013
}

\author{
|Regional Maternal and Child Hospital of Imperatiz, Maranhao: predominant \\ delivery pathway in Oetober and November 2013
}

\author{
Elen Diana Lopes Moraes Ribeiro ${ }^{1}$ \\ Iolanda Graepp Fontoura ${ }^{2}$ \\ Janayne Ribeiro Cordeiro ${ }^{3}$ \\ Paula Cristina Alves da Silva ${ }^{4}$ \\ Rodson Glauber Ribeiro Chaves ${ }^{5}$
}

\section{Resumo}

O parto é o estágio resolutivo da gestação, o nascimento do ser que se formou nos meses anteriores, pode ser pela via vaginal onde ocorre a expulsão do feto para o mundo exterior, ou através da operação cesariana, a retirada do bebê por via transabdominal. O presente estudo teve como objetivo a predominância de partos cesáreos e vaginais realizados no Hospital Regional Materno Infantil de Imperatriz-MA. Discorrer sobre à proporção que acontece esses tipos de partos no Hospital para conhecer a via de parto predominante. Pesquisa de campo, documental, quantitativa onde foi utilizada uma ficha de investigação para coleta de informações nos prontuários a fim de identificar a quantidade de partos total; a quantidade de partos cesáreos e vaginais e quais as indicações médicas para os partos cesáreos. Os resultados obtidos revelam que 60,4\% realizaram partos vaginais e $39,6 \%$ partos cesáreos, as indicações médicas mais frequentes para o parto cesáreo foram: parada de progressão (29,2\%); iteratividade $(13,1 \%)$ e sofrimento fetal $(11,9 \%)$. Diante do exposto, percebe-se que os profissionais de saúde devem atuar de forma a minimizar os fatores mutáveis às indicações de cesarianas e os riscos que acompanham essa via de parto para que também decresçam.

\section{Abstract}

Childbirth is the resolving stage of pregnancy, the birth of the being who graduated in the previous months, can be vaginally where the expulsion of the fetus to the outside world, or by caesarean section, the removal of the baby by transabdominal ultrasound occurs. The present study aimed to the prevalence of cesarean and vaginal deliveries in the Hospital Regional Materno Infantil in ImperatrizMA. Discuss what happens to the proportion of these types of deliveries at the hospital to know the predominant route of delivery. Fieldwork, documentary, where a quantitative investigation form was used to collect information in the records to identify the quantity of total births; the amount of cesarean and vaginal births and what the medical indications for cesarean deliveries. The results show that $60.4 \%$ had vaginal deliveries and cesarean deliveries $39.6 \%$, the most frequent medical indications for cesarean delivery were failure to progress $(29.2 \%)$; iterativity ( $13.1 \%$ ) and fetal distress ( 11.9 $\%)$. Given the above, it is perceived that health professionals should act to minimize the shifting factors for cesarean indications and risks that accompany this type of delivery to also progressively decrease.

Deseritores: Parto. Cesárea. Parto Normal.

Keywords: Delivery. Cesarean. Normal childhirth.

\footnotetext{
${ }^{1}$ Enfermeira. Universidade Federal do Maranhão - Centro de Ciêneias Sociais, Sauide e Teenologia.
}

2 Enfermeira. Mestra. Professora do Curso de Enfermagem da Universidade Federal do Maranhão - Centro de Ciêneias Sociais, Saúde e Teenologia. 3. Enfermeira. Universidade Federal do Maranhão - Centro de Ciêneias
Sociais, Saivide a Tecnolonia.

4. Enfermeira. Mestra. Professora do Curso de Enfermagem da Universidade Federal do Maranhão - Centro de Ciêneias Sociais, Saúde e Tecnologia.

5. Enfermeiro. Especialista em Saúde Píbliea. Professor do Curso de Enfermagem da Universidade Federal do Maranhão - Centro de Ciêneias Sociais, Saúde e Tecnologia.

Para correspondência:

Rodson Glauber Ribeiro Chaves

E-mail: rodson_ribeiro8@hotmail.com 
Introducã̃o

O parto é o estágio resolutivo da gestação, o nascimento do ser que se formou nos meses anteriores. Pode ser pela via vaginal onde ocorre a expulsão do feto para o mundo exterior, ou através da operação cesariana, a retirada do bebê por via transabdominal. Durante a gravidez e o parto, as mulheres vivenciam várias alterações físicas e emocionais e expressam, neste processo, valores e crenças, assim como se defrontam com a estrutura social e cultural dos profissionais dos serviços de saúde ${ }^{1,2}$.

O parto normal é método natural de nascer e, como tal, possui a proteção das forças da natureza. Se a mãe for jogada à própria sorte, em mais de $92 \%$ das vezes ela terá o seu filho sem problemas. A sua recuperação é imediata, pois, logo após o nascimento, poderá levantar-se e atender seu filho. As complicações próprias do parto normal são menos graves quando comparadas com aquelas advindas do parto cirúrgico. A amamentação do recém-nascido se torna mais fácil e, mais saudável a ele; a infecção hospitalar é muito menos frequente no parto normal ${ }^{3}$.

No entanto, o índice de parto cesáreo tem crescido especialmente em gestações sem indicações clínicas justificáveis para essa prática cirúrgica, o que tem mudado o cenário obstétrico. Dentro dos parâmetros mundiais, o Brasil é um dos países com maior prevalência de partos cesáreos, sendo que desde a década de 70 existe uma tendência a supervalorização desta via. Isso se deve ao fator do aprimoramento das técnicas cirúrgicas e do suporte médico no pré e pós-parto que valoriza o procedimento cirúrgico em detrimento das condições fisiológicas de parturição, nota-se a divulgação errônea da ideia de que a cesariana não representa riscos. Com isso, é comum perceber a escolha materna e profissional pelo parto cesáreo.

Neste sentido, a cesárea é uma intervenção cirúrgica originalmente realizada para reduzir o risco de complicações materna e/ou fetal durante a gravidez e trabalho de parto. Mesmo com o avanço na qualidade e segurança deste ato cirúrgico ofertado à gestante e seu filho nas situações de maior complexidade, essa prática não é sinônimo de ausência de riscos coadjuvantes ${ }^{5}$.

$\mathrm{O}$ aumento das cirurgias cesarianas, quase sempre é justificado nos prováveis benefícios para a saúde do recém-nascido e da mãe, como por exemplo, em situações de gestação patológica como descolamento prematuro de placenta, placenta prévia, parto com desproporção céfalo-pélvica verdadeira, eclampsia, dentre outras intercorrências obstétricas ${ }^{6}$.

Um estudo do Ministério da Saúde mostra que a realização de parto cesáreo aumentou no país de $38 \%$ em 2000 para 52,3\% em 2010. Este aumento ocorreu em todas as regiões, tendo o Nordeste um crescimento de $25,6 \%$ em 2000 para 44,3\% em 2010. Segundo a Organização Mundial de Saúde (OMS), o objetivo da assistência ao parto é manter mulheres e recémnascidos saudáveis, com o mínimo de intervenções médicas, buscando garantir a segurança de ambos. Essa organização preconiza que o total de cesáreas em relação ao número total de partos realizados nos serviços de saúde seja de $15 \%$. Todavia, este procedimento cirúrgico deve ser realizado apenas quando há riscos para a mãe ou para o bebê.

Há uma preocupação com as altas taxas de cesarianas existentes, pois se tem tornado um problema de saúde pública, principalmente nos países em desenvolvimento. As cesarianas não justificáveis estão associadas a um maior risco para mãe e criança, maior período de internação e assim, contribuindo para o aumento do custo em financiamento público à saúde. A programação do parto cirúrgico deve ser rigorosa, sendo necessário que a mulher compreenda as indicações e os riscos de uma cesárea desnecessária, para tanto, é imprescindível que as gestantes sejam conscientes dos riscos do parto cesáreo e os benefícios do nascimento pela via natural ${ }^{8,9}$.

Pois, estudos mostram que o parto cesariano está relacionado ao maior risco de mortalidade e morbidade materna, como hemorragias, infecções puerperais, embolia pulmonar, riscos anestésicos, entre outros. Para o recém-nascido $(\mathrm{RN})$, há maior probabilidade de ocorrerem distúrbios respiratórios, icterícia fisiológica, prematuridade iatrogênica, hipoglicemia, anóxia, entre outros. Além disso, o vínculo mãe-filho sofre interferência, o qual pode influenciar negativamente $\mathrm{o}$ aleitamento materno ${ }^{10}$.

Ainda nesse contexto, vale salientar que a opção das mulheres contribui para esse alto índice. Em muitos casos a gestante opta pelo parto 
cesáreo independentemente de suas condições clínicas obstétricas. As razões para essa escolha frequentemente permeiam pelo medo da dor e mudanças na musculatura do canal vaginal. A ideia é que a cesárea eletiva, agendada com antecedência, permitirá um parto sem dores, desde que recebam também fortes analgésicos depois da cirurgia. O outro conceito é que a anatomia e fisiologia da vagina e do períneo da mulher continuarão intactas com a cirurgia cesariana, enquanto que o parto vaginal produz perda acentuada da função do coito normal ${ }^{11}$.

Com isso, humanizar a assistência de enfermagem materna infantil é de vital importância porque garante à mulher o seu acesso ao pré-natal, assegurando-lhe uma assistência digna, uma gravidez segura e saudável, com as informações necessárias para que possa escolher com tranquilidade o local, o tipo de parto, os profissionais, o acompanhante, a posição de parição, entre outras, respeitando sempre a participação da família em todo esse processo ${ }^{12}$. Para tanto, o enfermeiro atua no pré-natal por meio de consulta de enfermagem e de atividades em grupo, com o objetivo de garantir o bom desenvolvimento das gestações, prevenir riscos e identificar as clientes com maior probabilidade de apresentar intercorrências durante a gestação, promovendo a saúde da parturiente e do neonato através do diagnóstico e cuidados de enfermagem ${ }^{12}$.

Este artigo teve como objetivo conhecer a via de parto predominante em outubro e novembro de 2013 no Hospital Regional Materno Infantil de Imperatriz- MA, contabilizar o número de partos totais nos meses de outubro e novembro de 2013, além de quantificar os partos vaginais e cesáreos e investigar a indicação clínica para os partos cesáreos.

Levando em consideração a situação descrita, considera-se relevante conhecer a prevalência de partos cesariana e vaginal e as indicações de cesarianas, pois acredita-se que esses dados possam contribuir para 0 acompanhamento das taxas no respectivo Hospital em estudo e demais. Diante dos resultados buscar-se-á novas estratégias capazes de reversão das altas taxas encontradas.

\section{Metodologia}

O estudo foi do tipo documental, com abordagem quantitativa, a partir de informações do prontuário. Realizado no Estado do Maranhão, situado na região nordeste do país, ocupa uma área de 331.935,507 $\mathrm{km}^{2}$. Limita-se ao norte pelo Oceano Atlântico, ao leste e sudeste com o Estado do Piauí, ao sul e sudeste com o Estado do Tocantins e a oeste com o estado do Pará. O Maranhão apresenta uma população de cerca de 6.574.789 habitantes distribuídos em 217 municípios, conforme censo demográfico 2010. A cidade de Imperatriz tem área de $1.368,987 \mathrm{~km}^{2} \mathrm{e}$ população de 247.505 habitantes. A cidade se estende pela margem direita do rio Tocantins e é atravessada pela Rodovia Belém-Brasília, situando-se na divisa com o estado do Tocantins ${ }^{13}$.

A presente pesquisa foi realizada com pacientes do Hospital Regional Materno Infantil de Imperatriz-MA, que está situado na Rua Coriolano Milhomem, $n^{\circ} 42$, Centro, ImperatrizMA. O Hospital é uma maternidade pública e de referência que atende a população de toda a região Tocantina. O hospital recebe gestantes de vários municípios onde é realizada uma quantidade elevada de partos todos os dias. Por atender casos de maior complexidade e em quantidade alta.

A população alvo do estudo constitui-se de mulheres atendidas neste hospital no período de outubro e novembro de 2013, totalizando 1.036, representadas a partir de seus prontuários. A escolha por esses respectivos meses deve-se pela frequência de partos, resultante do número de gestações ocorridas durante o período do carnaval, que popularmente chama-se de "filhos do carnaval". Para alcançar os objetivos foi utilizada uma ficha de investigação para a coleta de dados nos prontuários, os quais revelaram o número total de partos nesse período, o número de partos vaginal e cesáreo, além dos principais fatores que levaram as mulheres a realizarem o parto cesáreo.

Os dados coletados na pesquisa em prontuários foram armazenados em um banco de dados no programa Microsoft Office Excel ${ }^{\circledR} 2010$ e distribuídos estatisticamente sob forma de tabelas simples, com frequência absoluta e percentual, sendo analisados com base no referencial teórico estudado.

No tocante dos aspectos éticos, o trabalho foi encaminhado à instituição, acompanhado de ofício e do termo de fiel depositário solicitando a entrada no campo de pesquisa, a qual foi concedida, em documento, 
por seu dirigente.

A presente pesquisa foi submetida e aprovada pelo Comitê de Ética em Pesquisa do Hospital Universitário da Universidade Federal do Maranhão sob o registro $n^{\circ} 6011 / 2013$, sendo emitido o parecer favorável para realização da pesquisa em 03 de outubro de 2013.

\section{Resultados e diseussão}

A amostra total da pesquisa constituiuse de 1036 mulheres, que realizaram os partos na maternidade estudada. Destas, 626 realizaram parto vaginal nesses dois meses, com percentual de $60,4 \%$ e 410 mulheres realizaram parto cesáreo com percentual de 39,6\%. Durante o ano de 2013 a maternidade realizou um total de 6225 partos.

De acordo com os dados da Tabela 1, percebe-se que o valor percentual de partos vaginais ainda sobressai a quantidade de partos cesáreos, porém o índice de cesáreo é considerado superior ao preconizado pelo Ministério da Saúde e pela Organização Mundial de Saúde (OMS), os quais determinam respectivamente, até $25 \%$ e até $15 \%$ do número total de partos cesáreos realizados em um serviço de saúde ${ }^{14}$.

Um estudo sobre a incidência e características de cesáreas e partos normais na cidade de Baturité (Ceará) mostra que de janeiro 2003 a janeiro 2004, o percentual de cesáreas foi de $40,0 \%$. Essa taxa se deu pelas imprecisões nas indicações ou por conveniência profissional ${ }^{3}$.

No estudo realizado em hospitais brasileiros dos estados de São Paulo, Pernambuco e Distrito Federal por Pádua et al. (2010)15, revelou que a proporção de cesariana foi de $30,1 \%$. Nos hospitais de alta complexidade, a prevalência foi maior $(35,4 \%)$ do que nos de média complexidade (28,1\%). Esse estudo também mostrou que houve maior proporção de cesarianas naqueles hospitais em que só havia estudantes de medicina (39,2\%), e menor proporção nos que só havia estudantes de enfermagem/parteiras (26,7\%) e que nos hospitais de financiamento misto a proporção de cesárea foi de $32,0 \%$ enquanto nos que era financiado só pelo SUS o valor abaixou para $29,7 \%$.

O Hospital Regional Materno Infantil de Imperatriz-MA é referência em toda região no atendimento à maternidade, assistindo pacientes classificadas em baixo, médio e alto risco. A assistência baseia-se nas diretrizes norteadoras do Programa de Humanização no Pré-natal e
Nascimento e ainda possui o selo de hospital amigo da criança. Ainda assim, é perceptível a desproporção de cesáreas realizadas anualmente quando comparadas as taxas recomendadas pelo MS e OMS ${ }^{16}$.

Esse aumento de cesarianas nos últimos anos está relacionado ao aumento da segurança desse procedimento. Além disso, o parto cesáreo pode ser agendado, o tempo de cirurgia é menor em relação a um trabalho de parto normal, favorecendo assim o profissional médico ${ }^{17,18}$.

A visão panorâmica disposta por este estudo e demais na mesma linha revelam a proporção crescente de partos cesáreos em todo o Brasil com taxas mais altas do que as recomendáveis. Esse tipo de parto só deve ser escolhido mediante a indicação médica, pois se realizado indevidamente pode acarretar problemas para a mãe e para o bebê. De acordo com a tabela, nota-se que as taxas de cesáreas estão além dos parâmetros de uma maternidade da rede pública, onde o parto normal deveria ser mais incentivado e realizado.

\begin{tabular}{lcc}
\hline Via de parto & $\mathbf{N}$ & $\%$ \\
\hline Vaginal & 626 & 60,4 \\
Cesáreo & 410 & 39,6 \\
\hline TOTAL & 1036 & 100 \\
\hline
\end{tabular}

Tabela 1.Distribuição do número total de partos e a via de parto predominante, Imperatriz-MA, 2013.

Fonte: Pesquisa direta, 2013.

A Tabela 2 revela a divisão dos partos relacionados aos meses de outubro e novembro e a porcentagem de parto cesáreo e vaginal referente a cada mês. Respectivamente os partos vaginais obtiveram um percentual de $60,03 \%$ e $60,85 \%$, enquanto o parto cesáreo obteve $39,96 \%$ e $39,14 \%$.

Através dos dados representados na tabela 2, pode-se perceber que as taxas são basicamente as mesmas, sem muitas variações. $\mathrm{O}$ parto vaginal continua com índices por volta dos $60,0 \%$ sendo o tipo de parto que prevalece nesse estudo. No estudo de Cabral; Costa; Junior $(2003)^{19,20}$, a via de parto prevalente na maternidade Prof. Monteiro de Moraes em Recife$\mathrm{PE}$ foi à via vaginal com $72,3 \%$. Porém, a taxa de cesariana foi considerada alta em relação à 
recomendada e tem como justificativa a Maternidade ser reconhecida como instituiçãoescola de nível terciário, dotada de enfermaria para gravidez de alto risco e recebendo casos de alta complexidade ou graves.

Um estudo aplicado em São Luís-MA, em duas unidades hospitalares, uma pública e outra privada, teve como resultados $54,0 \%$ de partos pela via vaginal e $46,0 \%$ pelo procedimento cirúrgico no hospital público, porém, esses resultados mudam totalmente de valores, crescendo absurdamente as taxas de parto cesáreo para $97,8 \%$ no hospital privado. Nas duas maternidades, o principal motivo alegado para a preferência desse parto foi o fato de a realização ser mais rápida e para as mulheres que manifestaram preferência pelo parto cesáreo, o motivo foi o medo de sentir dor ${ }^{20,21}$.

A cesariana tem sido realizada de forma exagerada e crescente, voltando-se contra os objetivos para os quais foi idealizada. Com isso, ocasiona-se aumento nos riscos demorbimortalidade materna e perinatal, além da ausência de impacto na redução das taxas de perimortalidade ${ }^{22}$.

Estratégias têm sido voltadas para a redução das indicações, confirmando a informação de que as cesáreas não oferecem ganhos adicionais, aumentam os riscos maternos, oferecendo implicações para gestantes futuras e aumento de custos para os sistemas de saúde.

\begin{tabular}{lcccc}
\hline $\begin{array}{l}\text { Via de } \\
\text { Parto }\end{array}$ & \multicolumn{2}{c}{ Outubro } & \multicolumn{2}{c}{ Novembro } \\
\hline Vaginal & $\mathrm{N}$ & $\%$ & $\mathrm{~N}$ & $\%$ \\
Cesáreo & 217 & $326,96 \%$ & 193 & $39,14 \%$ \\
\hline Total & $\mathbf{5 9 3}$ & $\mathbf{1 0 0} \%$ & $\mathbf{4 9 3}$ & $\mathbf{1 0 0} \%$ \\
\hline
\end{tabular}

Tabela 2. Distribuição de partos vaginais e cesáreos nos meses de outubro e novembro de 2013, Imperatriz-MA, 2013.

Fonte:Pesquisa direta, 2013.

A Tabela 3 se encontram as indicações clínicas que levaram as mulheres a realizarem o parto cesáreo. Segundo os dados coletados nos prontuários, os principais diagnósticos encontrados foram: parada de progressão com $29,2 \%$, iteratividade com $13,1 \%$ e sofrimento fetal com $11,9 \%$.

Segundo o estudo de Yazlleet al. (2001)23 em seu estudo mostra que os principais diagnósticos associados aos partos cesáreos foram sofrimento fetal, cujas incidências foram 9,5\%, $10,9 \%$ e $9,0 \%$, respectivamente, nas categorias particular, pré-pagamento e SUS; e desproporção céfalo-pélvica com taxas de $5,8 \%, 6,5 \%$ e 3,9\%, respectivamente, nas mesmas categorias mencionadas. Já no estudo comparativo de cesariana entre um hospital público universitário e um hospital privado obtiveram a iteratividade como indicação mais frequente com $26,7 \%$ no hospital público e $36 \%$ na instituição privado. Como segunda causa de indicação tem-se as distorcias nos dois hospitais e o Sofrimento fetal referente à indicação mais frequente no Hospital Público com $18,2 \%{ }^{24}$.

\begin{tabular}{lcc}
\hline Indicações & N & $\%$ \\
\hline Parada de Progressão & 120 & 29,2 \\
Iteratividade & 54 & 13,1 \\
Sofrimento Fetal & 49 & 11,9 \\
Pré-eclâmpsia & 22 & 5,36 \\
Gestação Anômala & 15 & 3,65 \\
Desproporção Céfalo-pélvica & 14 & 3,5 \\
Oligodrâmnio & 12 & 2,92 \\
Pós- datismo & 11 & 2,68 \\
Cesária Anterior & 10 & 2,43 \\
Falha de progressão & 10 & 2,43 \\
Pélvico anterior & 8 & 1,95 \\
Prolapso de cordão & 5 & 1,21 \\
Apresentação Anômala & 2 & 0,48 \\
Descolamento prematuro da & 2 & 0,48 \\
placenta & & \\
Distócia & 2 & 0,48 \\
HIV + & 2 & 0,48 \\
Placenta Prévia & 1 & 0,24 \\
Má formação do USG & 1 & 0,24 \\
Feto macrossômico & 1 & 0,24 \\
Ameniorrexe & 1 & 0,24 \\
Gemelar & 1 & 0,24 \\
Cardiopatia & 1 & 0,24 \\
Sem respostas & 1 & 0,24 \\
\hline Total & & 100 \\
\hline & & \\
\hline
\end{tabular}

Tabela 3. Representação das indicações médicas para o parto cesáreo, Imperatriz-MA, 2013

Fonte: Pesquisa direta, 2013 
Dados divergentes dessa pesquisa, foram encontrados num estudo feito na Maternidade de referência do estado do Piaú obteve como indicações de cesárea mais frequentes: desproporção céfalo-pélvica (DCP) com $14,5 \%$, cesárea iterativa e pré-eclâmpsia com $6,5 \%$ cada e amniorrex prematura com $5,0 \%{ }^{24}$.

\section{Conelusão}

Considerando que mesmo o parto vaginal sendo a via predominante encontrada neste estudo, deve-se destinar uma atenção maior para a elevação percentual de partos cesáreos ocorridos no Hospital em estudo e em todo o Brasil, pois fica evidente o crescimento desse procedimento cirúrgico realizado muitas vezes desarbitrariamente, o que pode acarretar em maiores riscos a saúde da mulher.

Dessa forma, cabe aos profissionais de saúde buscar estratégias que possam minimizar os índices encontrados nesta instituição, além da conscientização dos profissionais obstetras, sendo necessária a aplicação de rigorosos critérios clínicos para a indicação da cirurgia cesariana como palestras educativas voltadas especificamente para esses profissionais. Assim, percebe-se que a melhor opção no momento para manter um equilíbrio na escolha do tipo de parto, está baseada na educação, tanto dos profissionais, quanto das mulheres gestante.

\section{Referêneias}

1. Reis SLS, Penteado CEM, Chatkin MN, Estrela MS, Porto PG, Munaretto, MM Parto normal X Parto cesáreo: análise epidemiológica em duas maternidades no sul do Brasil. Revista da AMRIGS, Porto Alegre, 53 (1): 7-10, jan.-mar, 2009.

2. Pires D, Fertonami HP, Conill EM, Matos, TA, Cordova FP, Mazur CS. A influência da assistência profissional em saúde na escolha do tipo de parto: um olhar sócio antropológico na saúde suplementar brasileira. Revista Brasileira Saúde Materna Infantil, Recife, 10 (2): 191-197 abr. / jun., 2010.

3. Queiroz MVO, Silva, NSJ, Jorge MSB, Moreira TMM. Incidência e características de Cesáreas e de partos normais: estudo em uma cidade no interior do Ceará. Revista Brasileira de Enfermagem.58(6):68791, nov-dez, 2005.

4. Sakae TM, Freitas PF, D'orsi E. Fatores associados a taxas de cesárea em hospital universitário. Revista de Saúde Pública. 43(3):472-80, 2009.

5. Patah LEM, Malik AM. Modelo de assistência ao parto e taxa de cesárea em diferentes países. Revista de Saúde Pública. 45 (1): 185-94, 2011.
6. Campana HCR, Pelloso SM. Levantamento dos partos cesárea realizados em um hospital universitário. Revista Eletrônica de Enfermagem, v. 09, n. 01, p. $51-63,2007$.

7. Brasil. Ministério da Saúde. Saúde Brasil 2011: uma análise da situação de saúde e de evidências. Secretaria de Vigilância em Saúde/MS. 2010.selecionadas de impacto de ações de vigilância em saúde.

8. Torres US, Moraes MS, Priuli RMA. O parto cesáreo e o sistema de saúde brasileiro: um estudo em um hospital escola, 2005.

9. Pinto ACM, Lima LC, Gomes MMFG, Arantes RC, Alberto SA. Parto cesáreo eletivo no Brasil uma análise dos fatores associados com base na pesquisa nacional de demografia e saúde (PNDS), 2006.

10. Carniel EF, Zanolli ML, Morcillo AM. Fatores de risco para indicação do parto cesáreo em Campinas (SP). Revista Brasileira de Ginecologia Obstétrica. 29(1):34-40, 2007.

11. Faundes A, Cecatti JG. A operação cesárea no Brasil. Incidência, tendências, causas, consequências e propostas de ação. Cadernos de Saúde Pública. Rio de Janeiro, 7 (2): 150-173. Abr/jun, 1991.

12. Marque FC, Dias IMV, Azevedo L. A percepção da equipe de enfermagem sobre humanização do parto e nascimento. Esc. Anna Nery R Enfermagem.10 (3). 439-47. Dez. 2006.

13. Brasil. Agência Nacional de Saúde Suplementar. O modelo de atenção obstétrica no setor de Saúde Suplementar no Brasil: cenários e perspectivas. Rio de Janeiro: ANS. 158 p, 2008.

14. Pádua KS, Osis MJD, Faúndes A, Barbosa AH; Filho OBM. Fatores associados à realização de cesariana em hospitais brasileiros. Revista Saúde Pública. 44 (1): 70-9, 2010.

15. Batista DC, Chiara VL, Gugelmin AS, Martins PD. Atividade física e gestação: saúde da gestante não atleta e crescimento fetal. Revista Brasileira de Saúde Materna Infantil. Recife, 3 (2): 151-158, abr./jun. 2003.

16. Silva PCA. Políticas Públicas de Humanização no Nascimento: Percepção da Parturiente quanto à Assistência de Enfermagem no Pré-Parto do Hospital Regional Materno Infantil de ImperatrizMA. 2013. 92f. Dissertação - [Mestrado].Pontifícia Universidade Católica de Goiás, Mestrado em Ciências Ambientais e Saúde; Goiânia, 2013.

17. Fonseca CC, Rocha LA. Gestação e atividade física: manutenção do programa de exercícios durante a gravidez. Revista Brasileira Ciências e Mov. 20(1): 111-121, 2012.

18. Costa SM, Ramos JGL. A questão das cesarianas. [s.l.], [s.n], 2005.

19. Cabral SALCS, Costa CFF, Júnior SFC. Correlação entre a Idade Materna, Paridade, Gemelaridade, Síndrome Hipertensiva e Ruptura Prematura de membranas e a Indicação de Parto cesáreo. RBGO. V.25, $n^{\circ} 10,2003$

20. Mandarino NR, Chein MBC, Júnior FCM, Brito LMO, Lamay, ZC, Nina VJS, Mochel E G, Neto JAF. Aspectos relacionados à escolha do tipo de parto: Um estudo comparativo entre uma maternidade pública e outra privada, em São Luís, Maranhão, Brasil. Cad. Saúde Pública. Rio de Janeiro, 25(7): 1587-1596. Jul, 2009. 
21. Bonfante TM, Silveira GC, Sakae TM, Sommacal LF, Fedrizzi EN. Fatores associados à preferência pela operação cesariana entre puérperas de instituição pública e privada. Arquivos Catarinenses de Medicina. Vol. 38, n.1, 2009.

22. Yazlle MEHD, Rocha JSY, Mendes MC, Patta MC, Marcolin AC, Azevedo GD. Incidências de cesáreas segundo fonte de financiamento da assistência ao parto. Revista Saúde Pública. 35 (2): 202-206, 2001.

23. Fabri RH, Silva HSL, Lima RV, Murta EFC. Estudo comparativo das indicações de cesariana entre um hospital público-universitário e um hospital privado. Ver. Brás. Saúde materna infantil.Recife, 2 (1): 29-35, jan - abril, 2002.

24. Feitosa GT, Assunção MS, Sousa PHS, Monte NL. A prevalência das vias de parto numa maternidade de referência do Estado do Piauí. Revista Interdisciplinar UNINOVAFAPI. V.5, n.4, p.13-18. Out-nov-dez, 2012. 